\title{
HEPATECTOMIA LAPAROSCÓPICA NO TRATAMENTO DAS METÁSTASES HEPÁTICAS
}

\author{
Marcel Autran C. MACHADO, Fabio F. MAKDISSI, Felipe A. Rocha de ALMEIDA, \\ Manoel LUIZ-NETO, Antonio Cavalcanti de A. MARTINS e Marcel C. C. MACHADO
}

\begin{abstract}
RESUMO - Descreve-se experiência inicial e resultados imediatos da hepatectomia laparoscópica no tratamento de metástases hepáticas em quatro pacientes. Uma paciente foi submetida a hepatectomia direita e três foram submetidas a bissegmentectomia 6-7. O tempo médio cirúrgico foi de 360 minutos e 4 dias de internação. Uma paciente apresentou fístula biliar autolimitada. Mortalidade operatória foi nula. Conclui-se que hepatectomia maior laparoscópica é factível, segura e apresenta excelentes resultados imediatos, com retorno precoce às atividades profissionais. Entretanto, ainda são necessários estudos com maior número de casos e trabalhos comparativos para se estabelecer limites e indicações deste procedimento em metástases hepáticas.
\end{abstract}

DESCRITORES - Neoplasias hepáticas. Metástase neoplásica. Hepatectomia. Laparoscopia.

\section{INTRODUÇÃO}

Nos últimos decênios as ressecções hepáticas tornaramse o método de eleição para o tratamento curativo das metástases hepáticas. As principais indicações para a ressecção de metástases hepáticas são as de origem colorretal e neuroendócrinas. O desenvolvimento das técnicas de ressecção hepática só atingiu o nível atual graças ao melhor conhecimento da anatomia segmentar do fígado e ao desenvolvimento de técnicas, reduzindo o sangramento intra-operatório e as alterações funcionais hepáticas. Este avanço tecnológico permitiu redução acentuada da morbidade e mortalidade operatórias. Atualmente, a mortalidade aceitável é inferior a 2\% em centros especializados.

O desenvolvimento da cirurgia laparoscópica ocorreu paralelamente. $\mathrm{O}$ aumento da experiência e o desenvolvimento de novos instrumentais resultaram na aplicação desta tecnologia nas ressecções hepáticas. Atualmente vivenciou-se o crescimento exponencial das indicações da videocirurgia nas ressecções hepáticas em diversos centros ${ }^{(1,4,5)}$.

O objetivo do presente trabalho foi descrever a experiência inicial e resultados imediatos da hepatectomia laparoscópica maior no tratamento de metástases hepáticas.

\section{MÉTODOS}

Quatro pacientes do sexo feminino, com metástases hepáticas, foram submetidas a ressecção hepática maior por laparoscopia entre maio e setembro de 2007. Três pacientes tinham metástases hepáticas de origem colorretal e uma metástase única e metacrônica de câncer de mama.

A idade média foi de 63 anos (56 a 75 anos). Uma paciente foi submetida a hepatectomia direita e três foram submetidas a bissegmentectomia 6-7 (Figura 1). A técnica da hepatectomia direita laparoscópica foi descrita anteriormente ${ }^{(3)}$. A técnica de bissegmentectomia 6-7 utilizada foi o acesso intra-hepático ao pedículo glissoniano do setor posterior direito ${ }^{(4)}$. Em resumo, o doente é colocado em posição supina, em decúbito lateral esquerdo, colocando-se o cirurgião entre as pernas do paciente. Pneumoperitônio com $\mathrm{CO}_{2}$ com pressão intra-abdominal de $12 \mathrm{~mm} \mathrm{Hg}$. São utilizados cinco trocartes: três de $12 \mathrm{~mm}$ e dois de $5 \mathrm{~mm}$. Três formas de se obter delimitação da área a ser ressecada foram utilizadas: dissecção e ligadura separada da tríade portal extra-hepática em um caso, acesso intra-hepático ao pedículo glissoniano correspondente em dois casos, e utilização da técnica de hemi-Pringle ${ }^{(2)}$ em um caso. Neste último caso, o pedículo portal é seccionado com grampeador endoscópico com carga vascular durante a secção do parênquima.

Ultra-sonografia intra-operatória foi usada em todos os casos, sendo com transdutor laparoscópico em dois casos (Figura 1A) e uso de transdutor tradicional com incisão auxiliar nos demais.

Uma vez determinado o tipo de ressecção e após o controle do pedículo e da veia hepática, procedeu-se à secção do parênquima com o uso combinado de bisturi bipolar, bisturi harmônico e grampeador endoscópico com carga vascular. A peça foi retirada por meio de incisão

Departamento de Gastroenterologia, Faculdade de Medicina da Universidade de São Paulo, São Paulo, SP.

Correspondência: Dr. Marcel Autran C. Machado - Rua Evangelista Rodrigues, 407 - 05463-000 - São Paulo, SP. E-mail: dr@drmarcel.com.br 


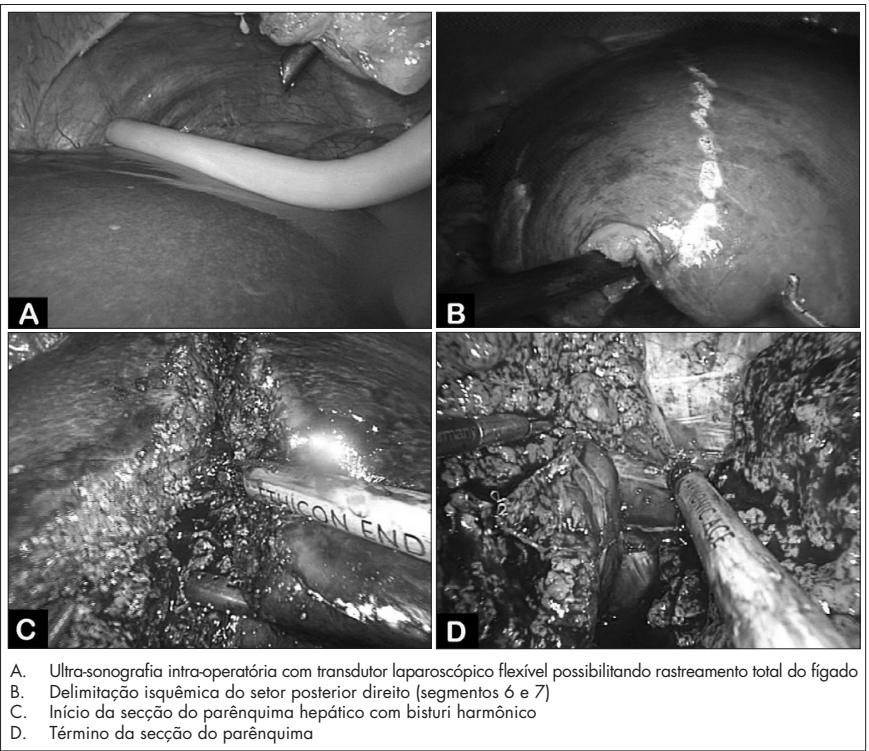

FIGURA 1. Bissegmentectomia 6-7 por laparoscopia

Pfannenstiel ou por meio de incisão sobre cicatriz pré-existente. Dreno tubular fechado foi colocado próximo à área cruenta.

Todas estas manobras foram feitas mantendo a circulação do fígado remanescente, isto é, não houve a necessidade de pinçamento total do hilo hepático (manobra de Pringle) em nenhum caso.

\section{RESULTADOS}

Todas as pacientes foram submetidas a hepatectomia laparoscópica com sucesso e sem necessidade de assistência manual. O tempo cirúrgico médio foi de 360 minutos ( 280 a 440 minutos). Uma paciente recebeu uma unidade de concentrado de hemácias durante $\mathrm{o}$ ato cirúrgico. $\mathrm{O}$ tempo médio de internação foi de 4 dias ( 3 a 5 dias). Uma paciente apresentou fístula biliar autolimitada com retirada do dreno com 14 dias. As demais tiveram seu dreno removido no $4^{\circ}$ dia de pós-operatório. Nenhuma paciente apresentou sangramento pelo dreno. Não houve necessidade de uso de auxílio manual ou conversão e a mortalidade operatória foi nula. $\mathrm{O}$ exame anatomopatológico demonstrou margens cirúrgicas livres em todos os casos.

\section{DISCUSSÃO}

Embora a ressecção das metástases colorretais seja rotina nos grandes centros, a ressecção laparoscópica dessas lesões não é comumente realizada. As razões para a lenta implantação da cirurgia mini-invasiva no tratamento das metástases hepáticas decorreram do receio do implante de células malignas e do temor de hemorragia incontrolável durante o procedimento. O desenvolvimento de novos instrumentais que possibilitam controle de grandes vasos e secção segura do parênquima hepático impulsionou o uso da laparoscopia nas ressecções hepáticas maiores. No entanto, a curva de aprendizado é longa, mas pode ser abreviada com o treinamento intensivo em animais de experimentação ${ }^{(3)}$, que parece reduzir as complicações técnicas quando do uso em humanos.

A comprovação de que o risco de implante neoplásico é mínimo, quando o procedimento segue os preceitos oncológi$\cos ^{(1,5)}$, associada aos excelentes resultados nos centros especializados, fez com que os autores desta casuística ampliassem as indicações deste procedimento para pacientes selecionados com metástases hepáticas.

A maior dificuldade atual em nosso meio, consiste na realização de ultra-sonografia intra-operatória. Em se tratando de metástase hepática, sabe-se que seu uso é imprescindível ${ }^{(2)}$. O motivo para tal reside na escassez de transdutores laparoscópicos próprios para este exame por via laparoscópica. Esses transdutores são onerosos e poucos hospitais dispõem deles. Em centros que não os possuem, uma alternativa é a introdução do transdutor cirúrgico tradicional por incisão auxiliar que será utilizada posteriormente para a retirada da peça.

Os resultados imediatos mostram que a ressecção laparoscópica é factível e segura. O sangramento intra-operatório foi mínimo em todos os casos e uma única paciente recebeu transfusão sangüínea.

A técnica empregada em nosso meio foi desenvolvida a partir de modelos experimentais em animais de médio porte ${ }^{(3)}$. $\mathrm{O}$ treinamento intensivo em animais fez com que a equipe reduzisse a curva de aprendizado e ganhasse experiência no controle de sangramento intra-operatório, que é a complicação mais temida neste tipo de procedimento. Outro benefício adquirido foi a adaptação de instrumentais disponíveis em nosso meio, o que possibilitou a realização, com sucesso, dos primeiros casos. Alguns instrumentais como o aspirador ultra-sônico, elemento chave para algumas equipes em outros centros e não disponível no Brasil para uso em videocirurgia, foi substituído por outros como, por exemplo, bisturi bipolar e bisturi harmônico.

Conclui-se que a hepatectomia maior laparoscópica é factível, segura e apresenta, apesar de casuística limitada, bons resultados imediatos, com recuperação rápida e retorno precoce às atividades profissionais. Seu uso em doenças benignas está bem estabelecido. No entanto, são necessário estudos com número maior de casos com seguimento tardio e trabalhos comparativos, para estabelecer os limites e as indicações deste tipo de procedimento em metástases hepáticas. 
Machado MAC, Makdissi FF, Almeida FAR, Luiz-Neto M, Martins ACA, Machado MCC. Laparoscopic hepatectomy for liver metastasis. Arq Gastroenterol. 2008;45(4):330-2.

ABSTRACT - The authors describe their initial experience and immediate results with laparoscopic liver resection in the treatment of four patients with liver metastasis. Three patients underwent laparoscopic anatomic bisegmentectomy 6-7 and one laparoscopic right hemihepatectomy. Mean operation time was 360 minutes and median hospital stay was 4 days. One patient developed bile leakage that was treated conservatively. No postoperative mortality was observed. This initial experience demonstrates that laparoscopic liver resection can be safely done with excellent immediate results. However larger and comparative studies are still needed to establish indications and limits for laparoscopy in the treatment of liver metastasis.

HEADINGS - Liver neoplasms. Neoplasm metastases. Hepatectomy. Laparoscopy.

\section{REFERÊNCIAS}

1. Koffron AJ, Auffenberg G, Kung R, Abecassis M. Evaluation of 300 minimally invasive liver resections at a single institution: less is more. Ann Surg. 2007; 246:385-92.

2. Machado MA, Makdissi FF, Bacchella T, Machado MC. Hemihepatic ischemia for laparoscopic liver resection. Surg Laparosc Endosc Percutan Tech. 2005; 15:180-3.
3. Machado MA, Makdissi FF, Surjan RC, Teixeira AR, Bacchella T, Machado MC. Hepatectomia direita por videolaparoscopia. Rev Col Bras Cir. 2007;34:189-92.

4. Machado MA, Makdissi FF, Galvão FH, Machado MC. Intrahepatic Glissonian approach for laparoscopic right segmental liver resections. Am J Surg. 2008;196:e38-e42.

5. Vibert E, Perniceni T, Levard H, Denet C, Shahri NK, Gayet B. Laparoscopic liver resection. Br J Surg. 2006;93:67-72.

Recebido em 7/11/2007. Aprovado em 27/2/2008. 